\title{
Allemagne : des réformes en perspective après PISA et IGLU
}

\author{
Le point sur...
}

Sandrine Lamer

\section{OpenEdition \\ Journals}

Édition électronique

URL : http://journals.openedition.org/ries/1612

DOI : $10.4000 /$ ries. 1612

ISSN : 2261-4265

Éditeur

Centre international d'études pédagogiques

Édition imprimée

Date de publication : 1 septembre 2003

Pagination : 17-19

ISBN : 978-2-85420-557-1

ISSN : $1254-4590$

Référence électronique

Sandrine Lamer, "Allemagne : des réformes en perspective après PISA et IGLU », Revue internationale d'éducation de Sèvres [En ligne], 33 | septembre 2003, mis en ligne le 12 avril 2012, consulté le 03 mai 2019. URL : http://journals.openedition.org/ries/1612 ; DOI : 10.4000/ries.1612

Ce document a été généré automatiquement le 3 mai 2019.

(c) Tous droits réservés 


\title{
Allemagne: des réformes en perspective après PISA et IGLU
}

\author{
Le point sur...
}

\section{Sandrine Lamer}

1 Alors que l'étude PISA ${ }^{1}$ 'a suscité en France qu'une réaction politique très modeste, elle est en Allemagne à l'origine d'une discussion intense qui remet en question le système éducatif allemand, dans son hétérogénéité comme dans son particularisme. Après PISA 2000, c'est PIRLS 2001 (Progress in International Reading Literacy Study, traduite par IGLU en Allemagne) qui alimente un discours de réforme.

2 PIRLS/IGLU est une évaluation internationale comparative de l'IEA (International Association for the Evaluation of the Educational Archievement) qui mesure les compétences de lecture au niveau de la quatrième année de primaire. C'est aussi bien la capacité de lire en tant qu'expérience littéraire que celle de lire à des fins d'acquisition et d'utilisation d'informations qui ont été testées. Tous les Bundesländer allemands y ont participé ; trente-cinq pays (dont la France) étaient également dans la compétition évaluative.

3 Si les résultats, parus au printemps 2003, ont placé l'Allemagne dans le premier tiers des pays participants, la chute entre les résultats plutôt satisfaisants obtenus par les enfants de dix ans (PIRLS/IGLU) et les résultats médiocres des enfants de quinze ans (PISA) inquiètent familles et spécialistes.

4 Pour les responsables en éducation, il faut tirer profit des leçons des études PISA, en particulier par rapport au classement des Bundesländer (paru en novembre 2002) et PIRLS/ IGLU. Rappelons que le système éducatif fédéral en Allemagne présente de grandes différences de fonctionnement selon les Länder, d'où l'intérêt d'une étude comparative interne, plus explicite quant aux forces et aux faiblesses du pays qu'une simple moyenne nationale. Les résultats de cette évaluation interne placent en tête la Bavière (légèrement en deçà de la Suède à l'échelle internationale), puis le Bade-Wurtemberg et la Saxe. Les trois mauvais élèves de l'Allemagne sont Brême (à l'échelle internationale entre le Portugal et le Luxembourg), en dernière position, puis le Brandebourg et la Saxe-Anhalt. À noter également que l'écart entre les résultats des premiers élèves et des derniers est 
très grand par rapport aux autres pays qui avaient participé à l'étude. Certes, c'est au niveau du Gymnasium, du fait des exigences de l'Abitur, que l'on trouve à échelle fédérale la plus grande homogénéité en références communes quant aux contenus et aux structures de l'enseignement dans les Länder. Et pourtant, de grandes divergences existent. Les trois premiers Bundesländer ont de nombreux points communs dans leur organisation du système éducatif. Par exemple le Zentralabitur est pratiqué comme forme d'évaluation de fin d'études du second cycle (baccalauréat allemand, centralisé pour tous les élèves des Gymnasien), alors qu'à Brême ou dans le Brandebourg, chaque établissement scolaire propose ses propres épreuves du baccalauréat à une commission régionale qui les valide.

5 «Malgré des résultats décevants, les études PISA et IGLU étaient très importantes puisqu'elles ont permis de créer une base empirique pour une nouvelle orientation stratégique des systèmes éducatifs $»^{2}$ a déclaré Andreas Schleicher, coordinateur mondial de PISA et lauréat 2003 du prix Theodor-Heuss pour son travail dans le cadre de l'étude. Grâce à lui en effet, un débat de grande envergure et de longue haleine sur le système éducatif, mûr depuis longtemps, a été lancé.

6 Le discours réformateur tenu par les représentants des partis politiques depuis la publication des résultats de l'étude PISA se concrétise en décisions et en actions. Karin Wolff, présidente de la KMK (Conférence permanente des ministres de l'Éducation et de la Culture), ne choisit pas une réforme sur la structure, mais sur la qualité de l'enseignement dispensé en Allemagne. Ainsi, la décision des actions à mener, prise le 6 mars 2003 à Berlin par la Conférence permanente suite aux résultats des évaluations, porte sur cinq points.

7 Tout d'abord, les sept mesures proposées par la KMK en décembre 2001 seront prises. Elles concernent l'amélioration des compétences linguistiques dès l'âge pré-scolaire ; une meilleure corrélation entre les structures pré-scolaires et primaires; l'amélioration de l'enseignement à l'école primaire et l'amélioration continue des compétences de lecture ainsi que de la mise en relation des mathématiques et des sciences pour une meilleure compréhension des liens interdisciplinaires; le soutien des élèves défavorisés, en particulier des enfants et des jeunes issus de l'immigration; le développement et la garantie de la qualité de l'enseignement sur la base de références de base communes et d'une évaluation régulière des résultats obtenus ; l'amélioration du professionnalisme des enseignants en particulier en matière de diagnostic et de méthodologie en tant qu'élément fondamental du développement de l'enseignement; la mise en place de la Ganztagsschule («l'école toute la journée ») avec une coopération des structures scolaires et para-scolaires. L'objectif de cette extension est de proposer un soutien scolaire et éducatif aussi bien aux plus défavorisés qu'aux élèves particulièrement doués.

Vient s'ajouter à ces mesures une volonté d'élargir l'enseignement à un apprentissage des compétences sociales. Karin Wolff a fait de «l'éducation par la famille et l'école» le thème central de son action en politique éducative.

9 Ensuite, l'hétérogénéité des profils des élèves, en particulier en fonction de leur milieu social et des problèmes spécifiques liés à l'immigration, nécessite une différenciation plus intense de l'enseignement et une individualisation renforcée du soutien offert aux élèves.

10 La Conférence permanente des ministres de l'Éducation et de la Culture publiera par ailleurs d'ici la fin de l'année 2003 des critères standardisés pour une plus grande homogénéité des actions de politique éducative régionale qui doivent conserver une 
partie de leur particularisme ainsi qu'un plan d'application. Les termes exacts de ces critères ne sont pas encore définis, les responsables politiques craignant vraisemblablement une confusion entre homogénéisation et centralisation. Ces références seront en corrélation directe avec la formation initiale et continue des enseignants.

Enfin, dans l'enseignement général comme dans l'enseignement professionnel, une plus grande transparence des systèmes scolaires doit apparaitre afin de mieux profiter des pratiques réussies des différents Länder.

Des actions sont d'ores et déjà lancées, comme le modèle de la Ganztagsschule associant établissements scolaires et structures culturelles et sociales (en Bavière, en Saxe, en BadeWurtemberg par exemple) ou le contrôle permanent des normes de qualité. En Thuringe, depuis février 2003, huit établissements scolaires participent à une évaluation modèle systématique de l'EFQM (European Foundation of Quality Management).

13 Ces réactions et décisions mettent en évidence une prise de conscience douloureuse des déficits du système actuel et, par voie de conséquence, un souci de travailler davantage sur la qualité de l'enseignement en Allemagne. Le fédéralisme n'est en soi pas remis en question: il est précisé qu'il permettra au contraire un échange de bonnes pratiques entre les Länder ainsi qu'entre les différents types d'établissements scolaires du second degré (Gymnasium, Gesamtschule, Realschule, Mittelschule), échange qui devrait assurer sur le long terme un suivi et une garantie de la qualité des enseignements dispensés, tant sur le plan des contenus que des méthodes.

\section{NOTES}

1. Programme international pour le suivi des acquis des élèves.

2. A. Schleicher, «La formation orientée vers l'avenir n'est pas une vision abstraite », GEW, p. 2, mai 2003.

\section{INDEX}

Mots-clés : réforme, éducation, PISA : programme international pour le suivi des acquis des élèves, IGLU, PIRLS : programme international de recherche en lecture scolaire

Index géographique : Allemagne 
AUTEUR

SANDRINE LAMER

Chargée de programmes, CIEP. 